\title{
In Retrospect: Brodmann's brain map
}

\author{
A classic neurology text written 100 years ago still provides the core principles for linking \\ the anatomy of the cerebral cortex to its functions today, explains Jacopo Annese.
}

\author{
Localisation in the Cerebral Cortex \\ by Korbinian Brodmann \\ First published 1909 (in German).
}

The development of advanced magnetic resonance imaging techniques over the past 30 years has heralded today's 'golden age' of human brain mapping. Yet in the quest to chart structural and functional subdivisions in the brain, and in the cerebral cortex in particular, the first quarter of the twentieth century was at least as momentous.

At the forefront of that pioneering effort was a German physician who is still revered by brain scientists today, but whose seminal book on cortical anatomy is read by few. Korbinian Brodmann's Localisation in the Cerebral Cortex, published 100 years ago, details his system for subdividing the cortex into 52 areas, each signified by a number, on the basis of their cell type and laminar structure. It is a classic of neurology: Brodmann's numbers are still used to indicate the location and function of cortical areas, and his trademark drawings of the human brain are reprinted wherever neuroscience is taught or practised. Personally, I have re-read the book carefully because my laboratory is engaged in producing a map of the cortical surface based on anatomical criteria, just like Brodmann, but using modern digital technology (see http://thebrainobservatory.ucsd.edu/brodmann.html).

In Brodmann's era, most cortical mapping took place in pathological laboratories that were linked to mental asylums and overseen by government bodies. But his effort started in an independent institute - the Neurobiological Laboratory in Berlin, supported and run by the neurologists Oskar Vogt and Cécile Vogt. The Vogts were mainly concerned with the architecture of myelinated nerve fibres, and how different areas of the cortex were linked to specific physiological functions, which they tested by stimulating the brains of animals. They brought in Brodmann to carry out a topographical analysis of the human cerebral cortex based exclusively on its cellular structure (the cytoarchitecture), using a staining method developed by their contemporary, Franz Nissl.

The task was theoretically straightforward, yet it occupied Brodmann for almost a decade.
Using a microscope designed for the purpose, he undertook meticulous examinations of cortical tissue from the brains of humans and many other mammals, the results of which enabled him to construct his map of the human cortex. The map looks simple, yet the book makes it clear it is based on a monumental analytical effort. His exquisite powers of observation and great attention to detail transform for the reader the tedium of scientific annotation into an exercise in anatomical voyeurism.

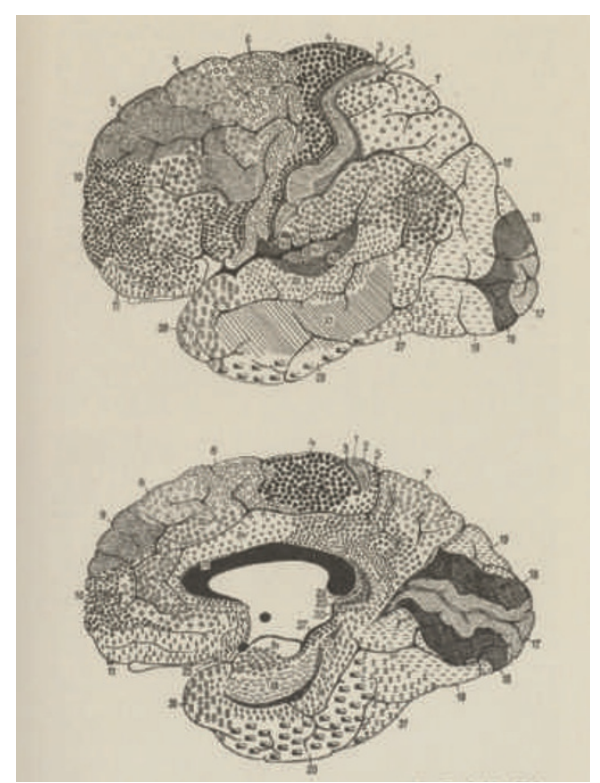

Two of Brodmann's classic drawings from 1909.

The descriptions alone mark out the book as a classic, but it is well worth reading today for several reasons. It touches on issues that are highly relevant to modern neuroscience, starting with the idea that specific physiological functions in the cerebral cortex depend on specific histological structure and connectivity.

Although Brodmann's numbers are widely used to link brain regions with function - area 4 for the primary motor cortex, for example - you get the sense from the book that he did not want to tarnish his mapping effort by making predictions about functional properties that might not stand the test of time. He reminded his readers that his investigation was based "exclusively on anatomical features", rather than on physiological or clinical approaches, although he does discuss these towards the end. Some of his peers were more forthright about labelling cortical regions according to function, notably the Australian-born neurologist A. W. Campbell, who used clinical evidence with results from physiological experiments and anatomical analysis to make his case. Still, Brodmann's objective approach has ensured that his maps have endured, eclipsing others of the time such as Campbell's.

Another of Brodmann's long-lasting achievements documented in the book is his defence of the accepted view that the cerebral cortex is divided into six fundamental layers, a view shared by some - but not all - neurologists of the time. Brodmann's rationale was based on his own interpretation of developmental and anatomical evidence that was potentially disputable, but his strong stance quieted the debate and it has been universally accepted with time. Indeed, the only valid criticism of Localisation in the Cerebral Cortex is that Brodmann failed to provide a clear photographic record of the cortical areas he was classifying. This technical drawback has made it hard to challenge or invalidate his conclusions about the way the cortex is subdivided, and has therefore preserved the impact of his map.

Brodmann's enterprise has stood up against what we have learned recently about brain architecture from modern neuroimaging techniques remarkably well. His approach remains convincing because it is based on a small number of unambiguous postulations and a deep personal involvement with the data. The arsenal of chemical and molecular techniques and digital-microscopy tools now at our disposal gives us far greater power to analyse brain structure than Brodmann had. Yet future projects should seek to learn from his impressive methods of synthesis rather than focusing solely on surpassing his methods. Whatever a new microscopic map of the cerebral cortex may look like, it will doubtless be based on the principles that Brodmann defended a century ago.

Jacopo Annese is director of The Brain

Observatory at the University of California,

San Diego, California 92121, USA.

e-mail: jannese@ucsd.edu

See online at go.nature.com/phyLwm for more on neuroscience. 